\title{
Optically sectioned in vivo imaging with speckle illumination HiLo microscopy
}

\author{
Daryl Lim, Tim N. Ford, Kengyeh K. Chu, and Jerome Mertz \\ Boston University, Department of Biomedical Engineering, 44 Cummington Street, Boston, Massachusetts 02215
}

\begin{abstract}
We present a simple wide-field imaging technique, called HiLo microscopy, that is capable of producing optically sectioned images in real time, comparable in quality to confocal laser scanning microscopy. The technique is based on the fusion of two raw images, one acquired with speckle illumination and another with standard uniform illumination. The fusion can be numerically adjusted, using a single parameter, to produce optically sectioned images of varying thicknesses with the same raw data. Direct comparison between our HiLo microscope and a commercial confocal laser scanning microscope is made on the basis of sectioning strength and imaging performance. Specifically, we show that HiLo and confocal 3-D imaging of a GFP-labeled mouse brain hippocampus are comparable in quality. Moreover, HiLo microscopy is capable of faster, near video rate imaging over larger fields of view than attainable with standard confocal microscopes. The goal of this paper is to advertise the simplicity, robustness, and versatility of HiLo microscopy, which we highlight with in vivo imaging of common model organisms including planaria, C. elegans, and zebrafish. @ 2011 Society of Photo-Optical Instrumentation Engineers (SPIE). [DOI: 10.1117/1.3528656]
\end{abstract}

Keywords: fluorescence; 3-D microscopy; optical sectioning; speckle; in vivo imaging.

Paper 10530R received Sep. 29, 2010; revised manuscript received Nov. 15, 2010; accepted for publication Nov. 30, 2010; published online Jan. 31, 2011.

\section{Introduction}

Fluorescence microscopy is commonly used to image biological specimens. The conventional wide-field fluorescence microscope provides high-resolution imaging for thin samples but suffers from poor contrast in thick samples due to the inevitable generation of background fluorescence in a standard Köhler illumination setup. The rejection of such background fluorescence leads not only to improved image contrast, but also 3-D resolution since the fluorescence intensity represented in the image is confined to a thin optical section. A variety of techniques that exhibit optical sectioning have been developed, the most ubiquitous being confocal laser scanning microscopy, often simply referred to as confocal microscopy. ${ }^{1}$ Many of these techniques work by shaping the illumination into a fixed pattern which results in an image that is inherently optically sectioned, or can be post-processed into an optically sectioned image. For example, confocal microscopy, two-photon excited fluorescence (TPEF) ${ }^{2}$ microscopy and other scanning illumination techniques rely on shaping the illumination into one or more focal spots. Other techniques such as structured illumination microscopy (SIM) and programmable array microscopy (PAM) depend on the generation of grid illumination at the focal plane, ${ }^{3,4}$ while sheet illumination techniques rely on confining a thin sheet of illumination to the focal plane. ${ }^{5,6}$

While these patterned illumination techniques each have their own advantages and are capable of producing excellent images under favorable conditions, their success invariably depends on the delivery of well defined and controlled illumination patterns into the sample. Recently, we have

Address all correspondence to Jerome Mertz, Boston University, Department of Biomedical Engineering, 44 Cummington St., Boston, MA 02215. Tel: 617-3580746; Fax: 617-353-6766; E-mail: jmertz@bu.edu. demonstrated a technique called dynamic speckle illumination (DSI) microscopy that produces fluorescence sectioning with illumination patterns that are neither well defined nor controlled. ${ }^{7-9}$ The idea of this technique is to illuminate a fluorescent sample with random speckle patterns obtained from a laser. Speckle patterns are granular intensity patterns that exhibit inherently high contrast. Fluorescence images obtained with speckle illumination are therefore also granular; however, the contrast of the observed granularity provides a measure of how in focus the sample is: high observed contrast indicates that the sample is dominantly in focus, whereas low observed contrast indicates it is dominantly out of focus. The observed speckle contrast thus serves as a weighting function indicating the in-focus to out-of-focus ratio in a fluorescence image. A key feature of speckle illumination is that while the exact intensity pattern incident on a sample is not known, the statistics of the intensity distribution are well known to obey a negative exponential probability distribution (provided the speckle is fully developed. ${ }^{10}$ ) According to this distribution, the contrast of a speckle pattern scales with average illumination intensity. Thus, weighting a fluorescence image by the observed speckle contrast is equivalent to weighting it by the average illumination intensity (as in standard imaging), however with the benefit that the weighting preferentially extracts only in-focus signal. A second key feature of speckle illumination is that its statistics are invariant even in a scattering medium, since unpredictable phase shifts induced by the medium only further randomize an already randomized laser phase front. Hence fluorescence sectioning based on speckle illumination is robust, since it is insensitive to scattering, aberrations, etc., in the illumination path.

In our original implementation of DSI microscopy, speckle contrast was measured by illuminating a sample with a series

1083-3668/2011/16(1)/016014/8/\$25.00 (C) 2011 SPIE 
of independent speckle patterns, and evaluating the variations in the observed speckle intensity over time. While effective, this technique proved to be slow since several images were required to obtain an accurate estimate of speckle contrast. A later implementation involved evaluating speckle contrast in space rather than time, using a single image. Such an evaluation inevitably entails a loss in spatial resolution; however, it was found that a second image obtained by standard uniform illumination could supply the missing high resolution information, ultimately resulting in a full (i.e., diffraction-limited) resolution, optically sectioned image from only two images. This technique is called HiLo microscopy. ${ }^{11-13}$

The goal of this paper is to demonstrate the unique advantages of HiLo imaging, using an improved algorithm from what was previously described and a more robust experimental layout, leading to significantly enhanced performance. We provide a direct comparison of HiLo microscopy with state-of-the-art confocal microscopy, and demonstrate a remarkable feature of HiLo microscopy that it provides a depth of field that can be fully tuned a posteriori (i.e., with the same set of two images). Moreover, we demonstrate for the first time in vivo imaging with real-time HiLo processing of exogenous and endogenous (GFP) labeled samples, in both high resolution microscopy and wide field of view (FOV) macroscopy configurations.

\section{Fundamentals of Speckle Illumination HiLo Microscopy}

A speckle illumination HiLo microscope is essentially a conventional wide-field fluorescence microscope with laser illumination and a digital camera (Fig. 1). The key difference lies in the introduction of a diffuser in the illumination path of the HiLo microscope. The diffuser randomizes the phase front of the laser beam, resulting in a speckle pattern which is projected into the sample via a microscope objective. One of the two

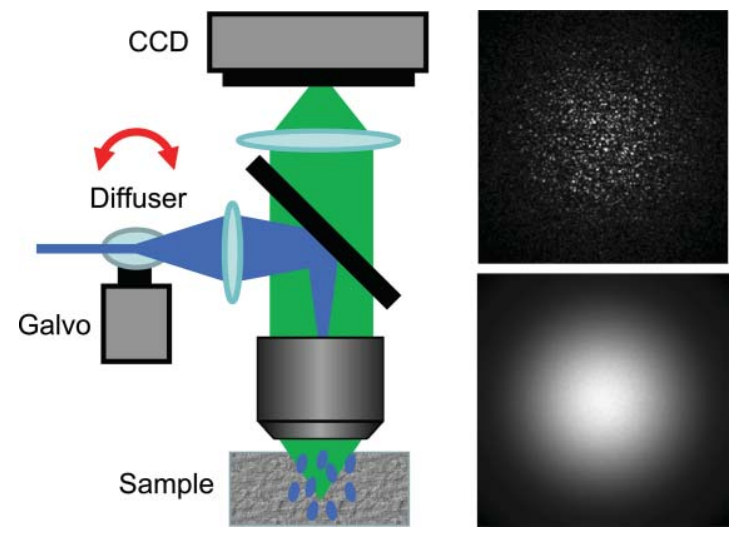

Fig. 1 A HiLo microscope setup is identical to a standard wide-field epi-fluorescence microscope except for the addition of a laser beam and diffuser in the illumination path, resulting in speckle illumination at the sample. The resulting fluorescence is captured by a digital camera. When the diffuser is static, the speckle illumination exhibits high contrast (top right panel). When the diffuser is rapidly oscillated by a galvanometric motor, the resulting speckle becomes blurred over the course of the camera exposure, effectively simulating uniform illumination (bottom right panel). Speckle and uniform illumination images are acquired pairwise, and processed into an optically sectioned HiLo image. images required for HiLo processing, $I_{s}$, is thus captured with speckle illumination. The second image, $I_{u}$, is captured with uniform illumination. To obtain the latter, we rapidly randomize the speckle pattern within a single exposure of the camera, effectively simulating uniform illumination. Randomization of the speckle pattern is most easily achieved by translating or rotating the diffuser. We have found that attaching a small diffuser to a galvanometer motor provides simple and reliable speckle randomization, with rapid response times that are crucial for high-speed imaging. The images acquired under speckle and uniform illumination are thus, respectively,

$$
\begin{gathered}
I_{s}\left(\vec{\rho}_{d}\right)=\iint \operatorname{PSF}_{\operatorname{det}}\left(\vec{\rho}_{d}-\vec{\rho}, z\right) O(\vec{\rho}, z) S(\vec{\rho}, z) d^{2} \vec{\rho} d z, \\
I_{u}\left(\vec{\rho}_{d}\right)=\iint \operatorname{PSF}_{\operatorname{det}}\left(\vec{\rho}_{d}-\vec{\rho}, z\right) O(\vec{\rho}, z)\langle S\rangle d^{2} \vec{\rho} d z,
\end{gathered}
$$

where $\operatorname{PSF}_{\operatorname{det}}(\vec{\rho}, z)$ is the 3-D detection point spread function of the microscope, $O(\vec{\rho}, z)$ is the 3-D object distribution, and $S(\vec{\rho}, z)$ is the 3-D speckle illumination intensity [note: in the case of uniform illumination, $S(\vec{\rho}, z)$ becomes randomized and averages to a constant $\langle S\rangle]$. For simplicity, we have assumed $\mathrm{PSF}_{\mathrm{det}}$ to be of unit magnification.

Equation (2) corresponds to a standard wide-field image. This image fails to exhibit optical sectioning because energy conservation requires $\int \operatorname{PSF}_{\mathrm{det}}(\vec{\rho}, z) d^{2} \vec{\rho}$ to remain constant, independent of $z$. That is, if $\operatorname{PSF}_{\operatorname{det}}(\vec{\rho}, z)$ is interpreted as a weighting function applied to $O(\vec{\rho}, z)$, this weighting function, globally, does not decay with defocus.

In contrast, we may look at the difference image $\delta I\left(\vec{\rho}_{d}\right)$ $=I_{s}\left(\vec{\rho}_{d}\right)-I_{u}\left(\vec{\rho}_{d}\right)$, given by

$$
\delta I\left(\vec{\rho}_{d}\right)=\iint\left[\operatorname{PSF}_{\mathrm{det}}\left(\vec{\rho}_{d}-\vec{\rho}, z\right) \delta S(\vec{\rho}, z)\right] O(\vec{\rho}, z) d^{2} \vec{\rho} d z,
$$

where $\delta S(\vec{\rho}, z)=S(\vec{\rho}, z)-\langle S\rangle$. The weighting function is now the term in square brackets. We find that this function does, globally, decay with defocus. Specifically, the larger the defocus, the broader $\operatorname{PSF}_{\operatorname{det}}(\vec{\rho}, z)$, and hence the more $\delta S(\vec{\rho}, z)$ becomes spatially averaged to zero. The decay to zero can be further accelerated by numerically applying an extra filter $W\left(\vec{\rho}_{d}\right)$ to the difference image, obtaining,

$$
\begin{aligned}
\delta I\left(\vec{\rho}_{d}\right)= & \iiint\left[W\left(\vec{\rho}_{d}-\vec{\rho}^{\prime}\right) \operatorname{PSF}_{\operatorname{det}}\left(\vec{\rho}^{\prime}-\vec{\rho}, z\right) \delta S(\vec{\rho}, z)\right] \\
& \times O(\vec{\rho}, z) d^{2} \vec{\rho}^{\prime} d^{2} \vec{\rho} d z
\end{aligned}
$$

The role of this extra filter will be made clear below.

As noted above, HiLo microscopy relies on a knowledge of the statistics of the speckle illumination pattern. For speckle that is fully developed and close to the optical axis, the transverse autocorrelation of the speckle variations is given by ${ }^{14}$

$$
\left\langle\delta S(\vec{\rho}, z) \delta S\left(\vec{\rho}^{\prime}, z^{\prime}\right)\right\rangle=\langle S\rangle^{2} A_{s} \operatorname{PSF}_{\mathrm{ill}}\left(\left|\vec{\rho}-\vec{\rho}^{\prime}\right|,\left|z-z^{\prime}\right|\right),
$$

where $\operatorname{PSF}_{\text {ill }}(\vec{\rho}, z)$ is the illumination point spread function, which, in general, may differ from $\mathrm{PSF}_{\text {det }}$, and $A_{s}$ $=\mathrm{PSF}_{\mathrm{ill}}(0,0)^{-1}$ roughly corresponds to the average transverse area of a speckle grain.

To understand how HiLo imaging performs optical sectioning, it is instructive to evaluate the contrast of the imaged speckle 
variations as a function of defocus. Specifically, let us consider a uniform plane object located at a defocus position $z$ (where $z=0$ is in focus). When this object is illuminated by speckle, the resulting granular fluorescence variations, upon imaging, exhibit a contrast given by

$$
C_{\delta s}(z)=\frac{\sigma_{\delta s}(z)}{\left\langle I_{s}\right\rangle}=\frac{\sigma_{\delta s}(z)}{\left\langle I_{u}\right\rangle},
$$

where $\sigma_{\delta s}(z)$ denotes the standard deviation of the imaged fluorescence variations. An evaluation of this contrast is more easily performed in frequency space. To this end, the optical transfer functions associated with the PSFs are defined to be

$$
\operatorname{OTF}\left(\vec{\kappa}_{\perp}, z\right)=\int \operatorname{PSF}(\vec{\rho}, z) e^{-i 2 \pi \vec{\kappa}_{\perp} \cdot \vec{\rho}} d^{2} \vec{\rho}
$$

and, similarly, $\mathcal{W}\left(\vec{\kappa}_{\perp}\right)$ is defined to be the Fourier transform of $W(\vec{\rho})$.

Finally, from Eqs. (4), (5), and (6) we arrive at

$$
C_{\delta s}^{2}(z)=A_{s} \int\left|\mathcal{W}\left(\vec{\kappa}_{\perp}\right)\right|^{2}\left|\mathrm{OTF}_{\mathrm{det}}\left(\vec{\kappa}_{\perp}, z\right)\right|^{2} \mathrm{OTF}_{\mathrm{ill}}\left(\vec{\kappa}_{\perp}, 0\right) d^{2} \vec{\kappa}_{\perp} .
$$

The above result is the basis of speckle illumination HiLo microscopy. As emphasized above, the imaged speckle contrast $C_{\delta s}(z)$ may be interpreted as a weighting function that is peaked when the object is in focus and decays to zero as the object goes out of focus. We observe that $C_{\delta s}(z)$ indeed obeys this property. The role of the extra filter $W(\vec{\rho})$ included in Eq. (4) also becomes apparent. For example, let us consider the case where the filter is absent (i.e., where $\mathcal{W}\left(\vec{\kappa}_{\perp}\right) \rightarrow 1$ ). It can be shown in this case that $C_{\delta s}(z)$ scales as $|z|^{-1}$ for large $z$. This scaling law is intermediate between the scaling laws prescribed by $\mathrm{OTF}_{\text {det }}\left(\vec{\kappa}_{\perp} \neq 0, z\right) \propto|z|^{-3 / 2}$ and $\operatorname{OTF}_{\text {det }}\left(\vec{\kappa}_{\perp}\right.$ $=0, z)=$ constant $^{15,16}$ If we introduce now a highpass filter such that $\mathcal{W}(\overrightarrow{0})=0$, thereby deleting the $\vec{\kappa}_{\perp}=0$ contribution in the integral, we find that $C_{\delta s}(z)$ obeys instead the improved scaling law $|z|^{-3 / 2}$. Moreover, by adjusting the width of this highpass filter, we can tune the width of the sectioning strength, as will be demonstrated below. In practice, $W(\vec{\rho})$ can be generalized to be a bandpass filter (previously termed a wavelet prefilter in Ref. 9).

A crucial assumption in the above evaluation of $C_{\delta s}(z)$ is that the contrast reflect the variations only in the imaged speckle and not in the object itself. In our previous HiLo implementation, we evaluated the local contrast of the speckle and uniform illumination images separately, and used the latter to correct for object-induced variations in the former. The more robust method introduced here is to evaluated the local contrast directly in the difference image. A low resolution estimate of the in-focus image is then constructed by applying a lowpass filter (LP) to the weighted uniform illumination image, obtaining $I_{\mathrm{LP}}\left(\vec{\rho}_{d}\right)=\mathrm{LP}\left[C_{\delta s}\left(\vec{\rho}_{d}\right) I_{u}\left(\vec{\rho}_{d}\right)\right]$. As described in Ref. 11, complementary high resolution information is constructed by applying a highpass filter $\operatorname{HP}\left(\vec{\kappa}_{\perp}\right)=1-\operatorname{LP}\left(\vec{\kappa}_{\perp}\right)$ directly to the uniform illumination image, obtaining $I_{\mathrm{HP}}\left(\vec{\rho}_{d}\right)=\operatorname{HP}\left[I_{u}\left(\vec{\rho}_{d}\right)\right]$. The final HiLo image is synthesized from the fusion of the above two images, resulting in

$$
I_{\mathrm{HiLo}}\left(\vec{\rho}_{d}\right)=\eta I_{\mathrm{LP}}\left(\vec{\rho}_{d}\right)+I_{\mathrm{HP}}\left(\vec{\rho}_{d}\right),
$$

where $\eta$ is a scaling function that ensures a seamless transition from low to high spatial frequencies. Specifically, $\eta$ compensates for the fact that the imaged speckle contrast is, in general, not equal to one even for objects that are in focus. From Eq. (8), we thus find $\eta=C_{\delta s}(0)^{-1}$. In practice, $\eta$ can be estimated $a$ priori based on a knowledge of the illumination and detection PSFs, or, alternatively, it can be inferred experimentally directly from $I_{\mathrm{LP}}$ and $I_{\mathrm{HP}}{ }^{11}$

So far, we have neglected the effects of noise in our algorithm. Shot noise and readout noise introduce additional fluctuations in $I_{s}\left(\vec{\rho}_{d}\right)$ and $I_{u}\left(\vec{\rho}_{d}\right)$, which in turn leads to a bias in the quantification of $C_{\delta s}^{2}$. However, this bias can be corrected for based on our a priori knowledge of the noise statistics. Specifically, for a camera gain $G$ and readout noise variance $\sigma_{r}^{2}$ the noise-induced bias is given by

$$
\sigma_{n}^{2}\left(\vec{\rho}_{d}\right)=\left(G\left\langle I_{s}\right\rangle+G\left\langle I_{u}\right\rangle+\sigma_{r}^{2}\right) \int\left|\mathcal{W}\left(\vec{\kappa}_{\perp}\right)\right|^{2} d^{2} \vec{\kappa}_{\perp} .
$$

The first two terms, $G\left\langle I_{s}\right\rangle$ and $G\left\langle I_{u}\right\rangle$, are variance contributions of shot noise. Filtering has the effect of reducing noise variance and is taken into account with the integral term. This bias must thus be subtracted from $\sigma_{\delta s}^{2}\left(\vec{\rho}_{d}\right)$ prior to the evaluation of $C_{\delta s}\left(\vec{\rho}_{d}\right)$.

We have also not considered the effects of pixelation in the $\mathrm{CCD}$ camera. If the pixel size is non-negligible compared to the size of the illumination or detection PSFs, then the additional filtering introduced by the pixels must be taken into account. This additional filtering can be incorporated in $W\left(\vec{\rho}_{d}\right)$.

The steps for obtaining $I_{\mathrm{HiLo}}\left(\vec{\rho}_{d}\right)$ are summarized below:

(1) Subtract $I_{u}\left(\vec{\rho}_{d}\right)$ from $I_{s}\left(\vec{\rho}_{d}\right)$ to form $\delta I\left(\vec{\rho}_{d}\right)$.

(2) Bandpass $\delta I\left(\vec{\rho}_{d}\right)$ with a user-defined filter $W\left(\vec{\rho}_{d}\right)$.

(3) Evaluate $C_{\delta s}^{2}\left(\vec{\rho}_{d}\right)$ according to Eq. (6).

(4) Remove the noise-induced bias from $C_{\delta s}^{2}\left(\vec{\rho}_{d}\right)$ by subtracting $\sigma_{n}^{2}\left(\vec{\rho}_{d}\right)$.

(5) Construct LP and complimentary HP filters (based on the choice of $W\left(\vec{\rho}_{d}\right)$ - see below).

(6) Evaluate $\eta$ according to Eq. (8).

(7) Evaluate $I_{\mathrm{HiLo}}\left(\vec{\rho}_{d}\right)$ according to Eq. (9).

\section{Materials and Methods}

All HiLo images were acquired with a home-built setup. A 491-nm diode-pumped solid state laser (50-mW Cobolt Calypso, leading to $4 \mathrm{~mW}$ at the sample) was used as a light source and a CCD camera (Qimaging Retiga-2000R) was used to detect the signal. Speckle illumination was generated with a small piece of diffuser (Luminit LSD) glued to the mirror of a galvonometer scanner (Sonima SM2804) placed roughly in a plane conjugate to the back aperture of the objective. Control and synchronization of the galvonometer and camera was carried out with National Instruments LabView.

The image processing algorithm was initially run postacquisition in Matlab (Mathworks) using the DIPimage toolbox (Technical University Delft). Subsequently the image processing was rewritten in CUDA-C to take advantage of the parallel computing architecture offered by graphical processing units (GPU). In particular, the GPU used was a NVIDIA GeForce GTX 280. This greatly shortened processing times, particularly for fast Fourier transforms, facilitating real-time processing of HiLo images. For example, the time taken to process a $1024 \times 1024$ image was shortened from $875 \mathrm{~ms}$ in Matlab 


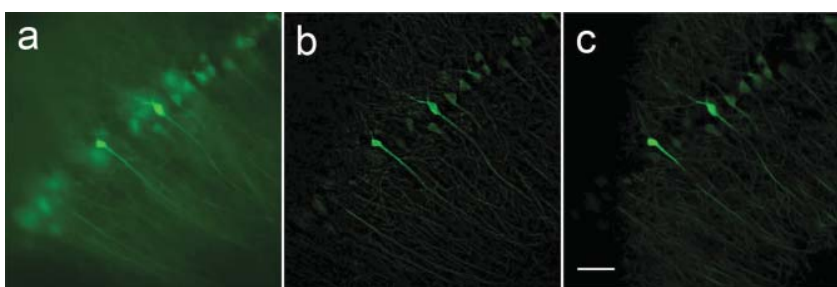

Fig. 2 Volumetric imaging of a mouse brain hippocampus slice (100 $\mu \mathrm{m}$ thick) labeled by cytoplasmic EGFP by in utero electroporation. ${ }^{17}$ $z$-stacks comprising 200 slices with $0.5 \mu \mathrm{m}$ step size were acquired with a HiLo microscope and a commercial confocal microscope (Olympus FluoView 1000), in both cases using a $20 \times$ water immersion objective (Olympus UApo/340). Co-located z-slices (single frames) presented in the left column were taken with (a) standard wide-field microscopy; (b) HiLo microscopy; and (c) confocal microscopy. HiLo exposure time was $2 \times 400 \mathrm{~ms}$ per slice (power limited with an illumination power at the sample of about $4 \mathrm{~mW}$ ), with images acquired at $1200 \times 1200$ resolution. Confocal exposure time was 2880 ms per slice (scan speed limited). Scale bar is $50 \mu \mathrm{m}$.

to $<40$ ms with our CUDA-C implementation. For the results presented below, in vivo videos were acquired and processed at $7 \mathrm{fps}$ (raw acquisition rate was $14 \mathrm{fps}$ ).

\section{Results}

Confocal microscopy remains the most common tool for obtaining background-free images. For HiLo microscopy to be broadly accepted by the bioimaging community, it is thus vital that HiLo and confocal microscopies be directly compared. To this end, we performed volumetric imaging of the same sample, namely cytoplasmic EGFP labeled mouse brain hippocampus, using both modalities. Single frames as well as extended focus images derived from a $z$ stack are presented in Fig. 2 and Video 1. As expected, conventional wide-field microscopy [Fig. 2(a)] suffers from the presence of background fluorescence, whereas both HiLo and confocal microscopy produced optically sectioned images of much higher contrast [Fig. 2(b), and 2(c)]. Extended focus images from the HiLo and confocal microscopes in Video 1 clearly reveal fine details in the neuronal arborization, despite the scattering nature of the sample. Background rejection enables not only improved resolution of neuronal structures but also 3-D reconstructions, where it is apparent that both techniques provide high-quality reconstructions that are largely identical.

A quantitative comparison of the background rejection capacity of HiLo and confocal microscopy can be obtained from sectioning curves (also called plane spread functions). A sectioning curve plots the integrated detected power produced by a thin, uniform, and in this case fluorescent, plane as a function of defocus, $z$. The profile of this function thus characterizes the sectioning strength of the imaging system. Using the same objective (Olympus LCPlanFL N) and sample (a thin, uniform film of FITC-albumin conjugate on a coverslip), we experimentally obtained sectioning curves for our home-built HiLo microscope (solid blue line) and a commercial state-ofthe-art confocal microscope (solid red line) shown in Fig. 3. Both systems demonstrate optical sectioning, as is manifested by the sharp decay in signal with defocus. Both curves exhibit similar widths, though the confocal curve has a marginally narrower full-width at half-maximum (FWHM), indicative of the slightly stronger sectioning expected by theory. The most distinctive difference between the two curves is the asymmetry and presence of sidelobes in the confocal sectioning curve. Such anomalies are well-documented and have been shown to be the result of spherical aberrations and the presence of a hard-edged confocal pinhole. ${ }^{18}$ These same effects are completely circumvented with HiLo microscopy since no physical pinhole is used

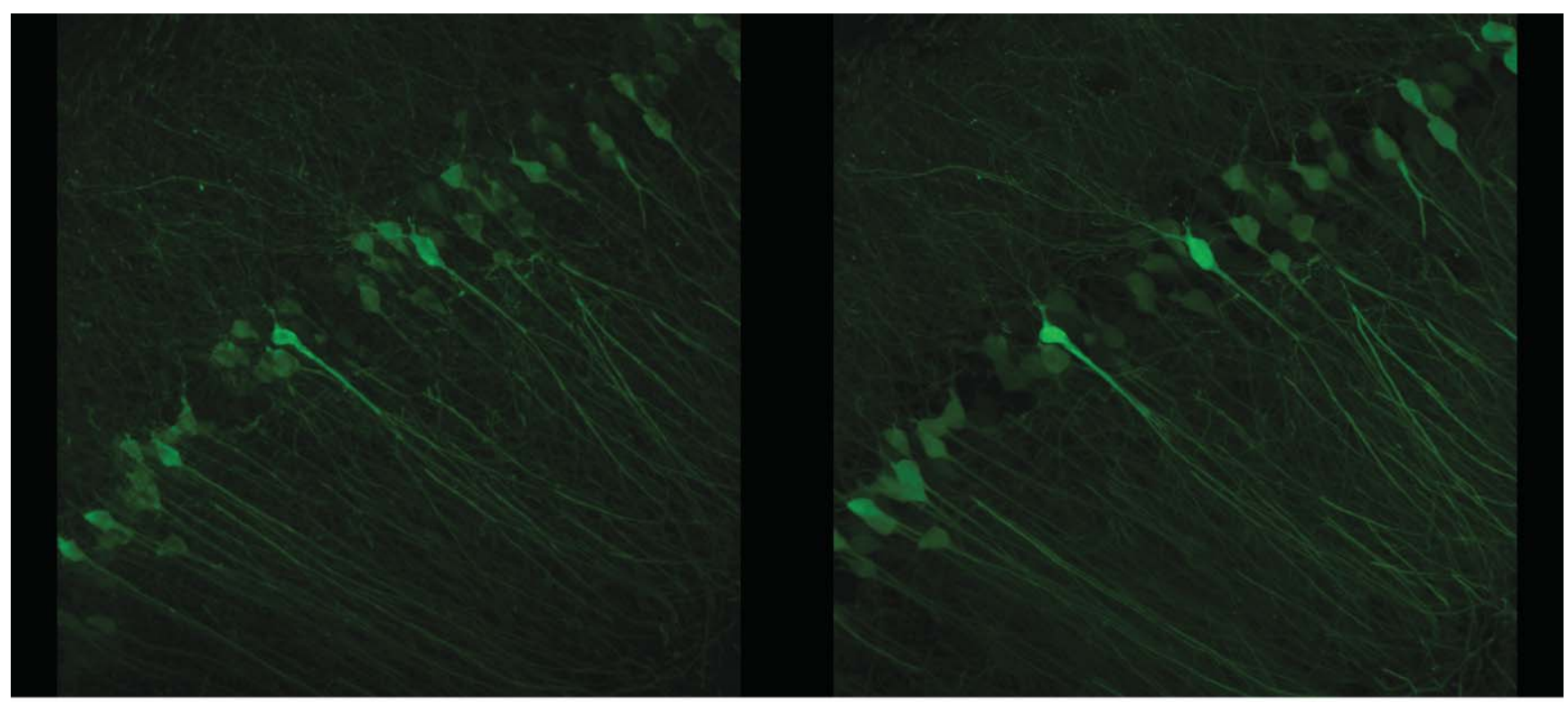

(a)

(b)

Video 1 Videos made from same imaging data as Fig. 2. (a) Maximum intensity projection of HiLo z-stack. (b) Maximum intensity projection of confocal z-stack. (MPEG, 9.4MB)

[URL: http://dx.doi.org/10.1117/1.3528656.1] 

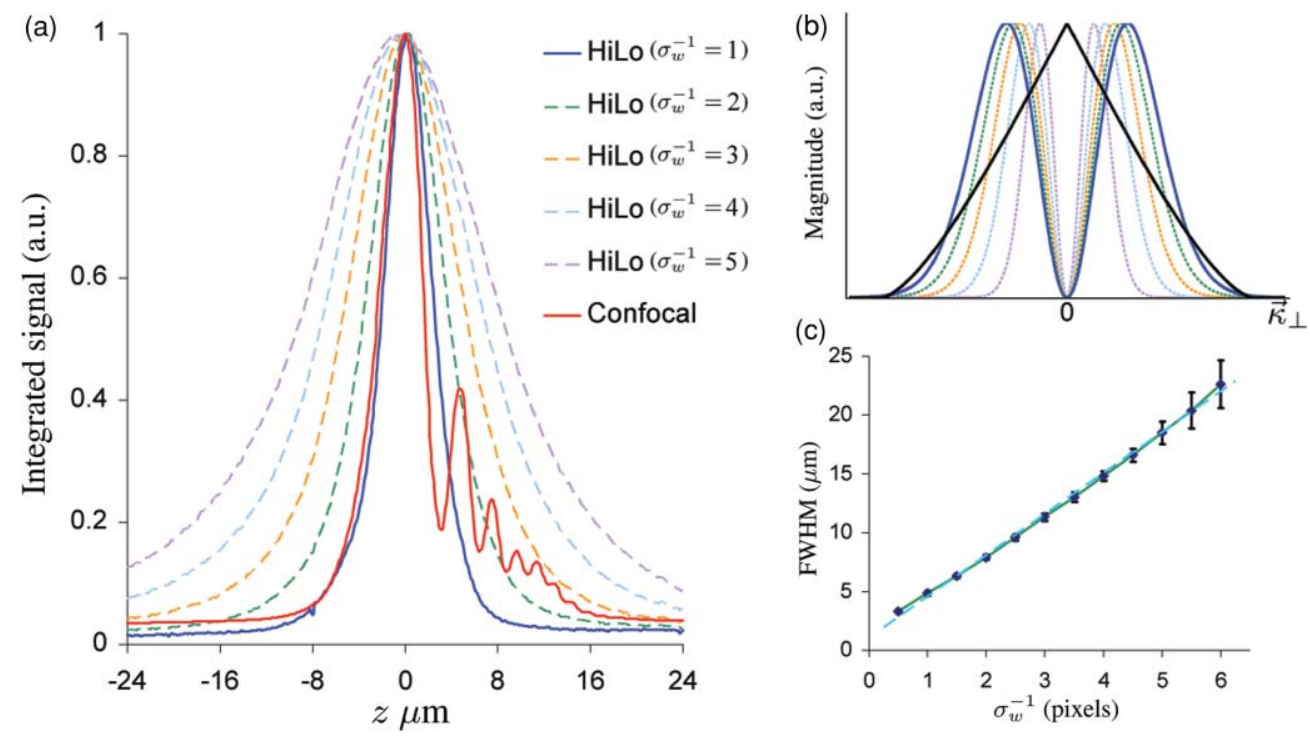

Fig. 3 (a) Confocal sectioning curve (solid red line) and HiLo sectioning curves processed with different $\sigma_{w}$ 's (solid blue and dashed lines) using the same data. (b) Bandpass filters used to generate HiLo sectioning curves (solid blue and dashed lines). Solid black line depicts the magnitude of the in-focus detection OTF for comparison. (c) FWHM (averaged over nine measurements) of HiLo sectioning curves as a function of $\sigma_{w}^{-1}$. Dashed cyan line indicates a linear fit $\left(R^{2}=0.998\right)$.

and also because the statistics of speckle illumination are largely unaffected by spherical (or any other) aberrations.

As described in the previous section, the sectioning ability of a HiLo microscope can be tuned by adjusting the bandpass filter $\mathcal{W}\left(\vec{\kappa}_{\perp}\right)$. For example, we use a bandpass filter defined in frequency space by the difference of two Gaussians:

$$
\mathcal{W}\left(\vec{\kappa}_{\perp}\right)=\exp \left(-\frac{\kappa_{\perp}^{2}}{2 \sigma_{w}^{2}}\right)-\exp \left(-\frac{\kappa_{\perp}^{2}}{\sigma_{w}^{2}}\right) .
$$

Such a filter is specified by a single parameter $\sigma_{w}$ and fulfills the criterion that $\mathcal{W}(\overrightarrow{0})=0$ [Fig. 3(b)]. A numerical evaluation of Eqs. (8) and (11) reveals that the FWHM of a HiLo sectioning curve varies inversely with $\sigma_{w}$, as confirmed by experiment. Thus the thickness of a HiLo optical section is linearly proportional to $\sigma_{w}^{-1}$ [Fig. 3(c)]. Since $\mathcal{W}\left(\vec{\kappa}_{\perp}\right)$ is applied only post-acquisition, the same pair of raw images can be used to generate many different HiLo images of varying axial thicknesses. This unique property of HiLo microscopy is illustrated in Fig. 3(a), where the dashed lines indicate different sectioning curves derived from the same raw data. However, the selection of $\sigma_{w}$ has repercussions on exactly how we must fuse the low and high frequency image content in $I_{\mathrm{LP}}$ and $I_{\mathrm{HP}}$. Indeed, by adjusting $\mathcal{W}\left(\vec{\kappa}_{\perp}\right)$, we are adjusting the axial thickness of $I_{\mathrm{LP}}$ only. To ensure that the axial thickness of $I_{\mathrm{HP}}$ is commensurate with that of $I_{\mathrm{LP}}$, we must also adjust the cutoff frequency separating these. The relationship between the axial resolution of $I_{\mathrm{HP}}$ and its spatial frequency content is described by the 3-D OTF of a widefield microscope, which can be approximated by an empirically derived expression known as the Stokseth approximation. ${ }^{15}$ Based on this approximation, we find that the cutoff frequency separating $I_{\mathrm{LP}}$ and $I_{\mathrm{HP}}$ should be set to approximately $0.18 \sigma_{w}$

Thus, the axial resolution (depth of field) of HiLo microscopy can be tuned a posteriori by varying a single parameter: $\sigma_{w}$. We demonstrate such tuning in Video 2, in which the same raw uniform and speckle images are processed into optically sectioned
HiLo images of varying thicknesses. It should be noted that while a thinner optical section results in better image contrast, a loss of depth of field is not always desirable. This can be a problem, for example, in confocal microscopy where the user
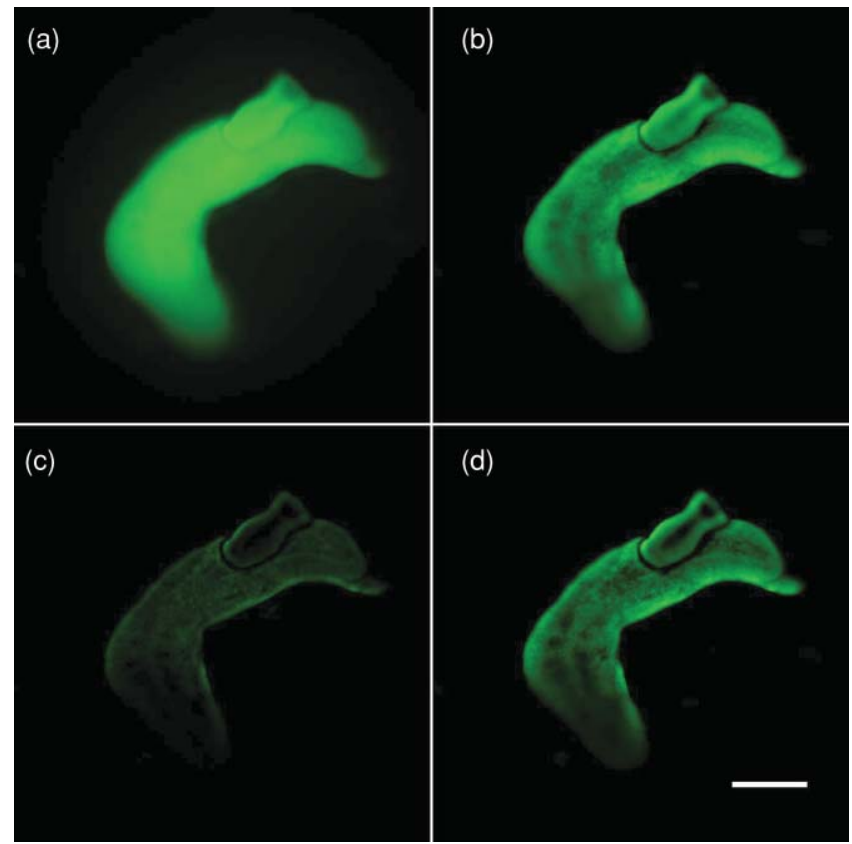

Video 2 Macroscopic HiLo videos of a live white planarian labeled by immersion with fluorescein diacetate (Sigma-Aldrich). All panels were generated from the same two raw images. (a) uniform illumination image. (b), (c), and (d) bottom left are HiLo images with decreasing depths of field, post-processed using filters with (b) $\sigma_{w}^{-1}=4$, (d) 2, and (c) 1 pixels respectively. Images were acquired with a $4 \times$ macroscope objective (Olympus MVX PLAPO 2XC), a 600×600 pixel resolution, and an exposure time of $2 \times 60 \mathrm{~ms}$ with an illumination power of about $4 \mathrm{~mW}$ at the sample. The scale bar is $0.4 \mathrm{~mm}$. (MPEG, 6.9MB) [URL: http://dx.doi.org/10.1117/1.3528656.2] 


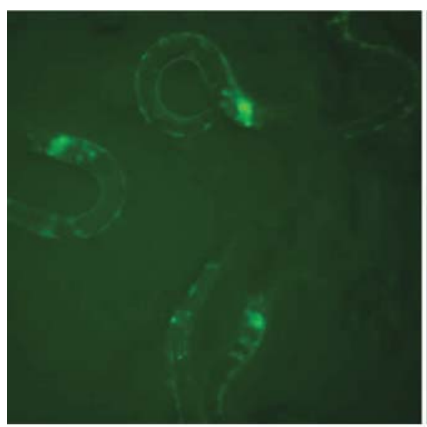

(a)

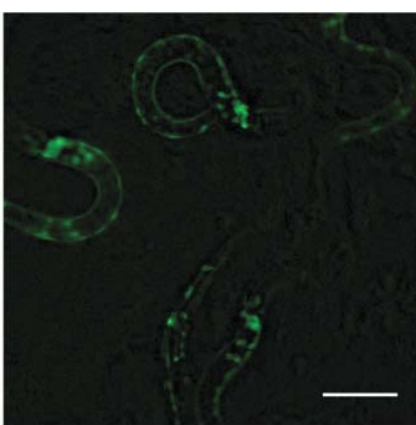

(b)

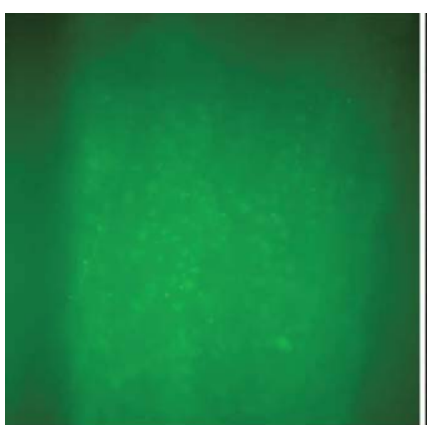

(a)

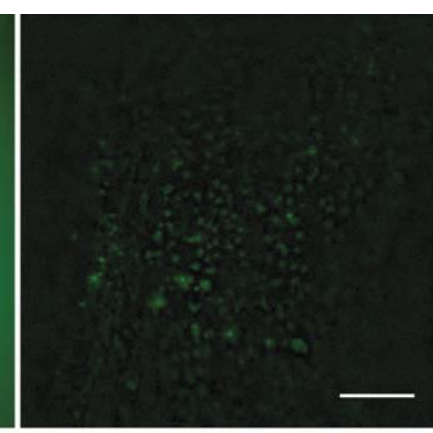

(b)

Video 3 In vivo imaging video. (a) Uniform illumination and (b) HiLo microscopic images of live $C$. elegans genetically encoded to express the calcium indicator cameleon in a specific subset of locomotor motorneurons. Images were acquired with a $40 \times$ microscope objective (Olympus LCPlanFL N), $600 \times 600$ pixel resolution, and an exposure time of $2 \times 60 \mathrm{~ms}$ with an illumination power of about $4 \mathrm{~mW}$ at the sample. The scale bar is $40 \mu \mathrm{m}$. (MPEG, 10MB) [URL: http://dx.doi.org/10.1117/1.3528656.3]

is constrained to a fixed depth of field for a given objective and pinhole size. While the depth of field in confocal microscopy can be increased by using a larger pinhole, such a solution is usually unappealing since it leads to poorer lateral resolution and requires the acquisition of new data. In contrast, the lateral resolution of a HiLo microscope is independent of $\sigma_{w}$, meaning no such trade-off between depth of field and lateral resolution is required.

We have demonstrated that HiLo microscopy provides 3-D imaging comparable to confocal microscopy; however, key performance requirements for biological imaging are not just image quality but also speed and FOV. These last two requirements are particularly important when imaging large, dynamic samples, such as live animal models. In addition to the planaria models shown in Video 2, we demonstrate in vivo HiLo imaging in other commonly studied animal models, namely C. elegans and zebrafish. These not only illustrate the versatility of HiLo microscopy, but also further highlight some of its advantages over confocal microscopy.

To begin, C. elegans are especially well-suited for imaging applications because they are optically transparent and can be readily studied fully intact. For example, due to its simplicity, the $C$. elegans nervous system is well-characterized with each neuron identifiable by position. Yet despite the benefits of optical sectioning conferred by scanning techniques such as confocal or TPEF microscopy, $C$. elegans are most often imaged with conventional wide-field microscopes for ease of use and generally superior imaging speed. ${ }^{19}$ HiLo microscopy provides a useful alternative to such conventional wide-field techniques, offering both the advantages of (tunable) optical sectioning and high imaging speed.

Video 3 shows live $C$. elegans in an agarose gel. The worms are labeled by cameleon, a genetically encoded calcium indicator consisting of both a cyan fluorescent protein (CFP) and yellow fluorescent protein (YFP). ${ }^{20}$ While cameleon indicators are normally used with YFP/CFP ratiometric imaging, we look only at the CFP emission here and the cameleon serves only as a fluorescent neuron labeler. The labeled neurons are mo-

Video 4 In vivo imaging video. (a) Uniform illumination and (b) HiLo macroscopic images of live zebrafish labeled with EGFP driven by a CD41 promoter, and anaesthesized with Tricaine. Images were acquired with a $4 \times$ macroscope objective (Olympus MVX PLAPO 2XC), a $600 \times 600$ pixel resolution, and an exposure time of $2 \times 40 \mathrm{~ms}$ with an illumination power of about $4 \mathrm{~mW}$ at the sample. The scale bar is $0.4 \mathrm{~mm}$ ). (MPEG, 10MB)

[URL: http://dx.doi.org/10.1117/1.3528656.4]

torneurons found mostly along the body but also in the head and tail. The HiLo images demonstrate a marked improvement in contrast over the conventional wide-field images (note the relative absence of fluorescence background generated from the thick agarose gel). Moreover, despite the rapid motion of the C. elegans, motion artifacts are largely absent in the HiLo images owing to the short camera exposure times $(2 \times 60 \mathrm{~ms})$, moderately high frame rate (net $7 \mathrm{~Hz}$ ) and simultaneous acquisition of all pixels across the FOV (Video 3).

High resolution in vivo imaging typically poses a number of challenges in addition to a requirement of speed. For example, a wide FOV is often desirable for the study of long range cellular dynamics. As will be discussed below, wide FOV imaging is particularly difficult to achieve with standard confocal microscopy. HiLo microscopy, however, is entirely compatible with wide FOV imaging. To demonstrate this, our home-built setup was designed to accommodate, besides conventional objectives, a macroscope objective that provides a large FOV and a large working distance, while maintaining high collection efficiency and resolution. Such a macroscope configuration was used in the in vivo imaging of planaria (Video 2) and zebrafish (Video 4).

Zebrafish are a common choice of animal model for the study of vertebrate development, again largely because of their relative optical transparency. While this property of transparency was previously limited to developing zebrafish, a mutant line casper has been introduced that exhibits almost entirely transparent adult bodies. ${ }^{21}$ We imaged live zebrafish (7 post-fertilization) with EGFP labeling driven by a CD41 promoter. Time-lapse imaging of such zebrafish are used in hematopoiesis studies to monitor the dynamics of CD41-low and CD41-high cells. ${ }^{23}$ The suppression of background fluorescence (Video 4) highlights EGFP-positive cells including thrombocytes and caudal hematopoietic tissue, which appear as circulating and static structures, respectively, in the video. Once again, we emphasize that motion artifacts are observed here to be minimal and that the FOV $\left(2.2 \times 2.2 \mathrm{~mm}^{2}\right)$ is much larger than could be attained with conventional confocal microscopy. 


\section{Discussion}

We have demonstrated that HiLo microscopy is capable of producing optically sectioned images comparable in quality to a commercial confocal microscope. This is achieved by processing two images, one taken with speckle illumination and another with conventional uniform illumination. An immediate advantage of this acquisition scheme is that for every backgroundfree HiLo image, the corresponding standard wide-field image is also available. Indeed, we have shown that by choice of a single post-processing parameter, the user can tune the level of background-rejection from confocal-like to completely unsectioned.

Because the raw images are acquired in wide-field mode, the limiting factors to speed are generally frame rate and illumination power. Randomization of the speckle by the galvanometer is currently not a limiting factor, since this has proven to be fast and reliable, producing stable performance at acquisition speeds beyond $50 \mathrm{fps}$. The numerical processing of images with our HiLo algorithm is also not a limiting factor, and is done in real time (see Sec. 3). Our prototype HiLo microscope currently operates at a speed of $7 \mathrm{fps}$ (for $600 \times 600$ images), limited by the frame rate of our CCD camera. In principle, given sufficient illumination power, HiLo microscopy should be able to operate at half the frame rate of even faster cameras. A faster camera would lead not only to faster imaging but also to a reduction in potential motion artifacts. Ideally, the two raw images should be acquired on a time scale more rapid than the sample dynamics of interest. However, even when this is not the case, HiLo microscopy remains fairly resistant to motion artifacts since all the high spatial-frequency information, in which motion is mostly captured, is extracted solely from the single image $I_{u}$. This is a significant departure from SIM, wherein high spatial frequency information becomes equally distributed among three raw images, leading to a greater susceptibility to motion artifacts. ${ }^{12}$ Because HiLo microscopy requires only two raw images, it can also conveniently be used with a double-shutter camera (commonly used for particle image velocimetry) to further reduce the time lapse between the recordings of $I_{u}$ and $I_{s}$.

For comparison, frame rates beyond $1 \mathrm{~Hz}$ in confocal microscopy generally require complex scanning mechanisms. For example, one configuration involves the use of a Nipkow disk to rapidly scan the illumination beam (or beamlets) across the sample. This can suffer from poor light efficiency and crosstalk between the multiple pinholes. ${ }^{1}$ Another approach involves the use of resonant galvanometer mirrors. Such mirrors utilize open-loop control and are capable of faster scan speeds than their closed-loop kindred. However, the trade-off is a loss of control of the scan speed that results in nonuniform sampling. ${ }^{22}$ Yet another strategy replaces the galvanometer-based scanning system with a rotating polygonal mirror capable of very high scan speeds at the cost of pointing precision. ${ }^{23}$ In the end, because of the technical complexities involved in fast imaging, the benefits of confocal microscopy are often trumped by the simple convenience of standard wide-field imaging with high frame rate $\mathrm{CCD}$ or CMOS cameras, which have the advantage of simultaneous sampling across the entire FOV.

With regard to FOV, this too can be compared between HiLo and confocal microscopy. Since the illumination beam is scanned in a confocal microscope, an increase in FOV would require not only an increase in scan range but also an expansion of the beam width to accommodate the large aperture of typical macroscope objectives. The requirements of large scan range and large beam expansion are inherently incompatible and impossible to achieve with standard galvonometer-based scanners, and prescribe instead the use of large galvonometer mirrors that would inevitably compromise imaging speed. Thus, in practice, fast confocal microscopes are typically limited to FOV's less than $0.5 \mathrm{~mm}^{2}$. As we have shown here, much larger FOV's can be readily achieved with HiLo imaging with minimal setup modifications. In this regard, HiLo imaging should prove to be especially useful in applications such as intravital microscopy, ${ }^{24}$ which would benefit from fast, optically sectioned imaging with large FOV's.

However, HiLo microscopy also has drawbacks compared to confocal microscopy. Specifically, background is rejected by a physical pinhole in confocal microscopy, whereas in HiLo microscopy (as in any post-processing technique such as SIM or PAM), it is rejected only numerically. As a result, the cameras used in HiLo microscopy must possess a higher dynamic range than required for confocal microscope detectors. Moreover, any shot noise associated with the background, while absent in confocal microscopy, must be corrected for in HiLo microscopy (albeit imperfectly — only a bias resulting from the shot noise can be corrected and not the random variations about this bias). Despite these drawbacks of post processing, HiLo microscopy remains nevertheless competitive with confocal microscopy, and, in many cases, advantageous, as shown above.

The crux of HiLo microscopy lies in the extraction of detected illumination contrast to distinguish in-focus signal from out-of-focus background. It should be noted that the source of illumination contrast is not limited to speckle. HiLo microscopy can be operated with essentially any high contrast pattern. For example, HiLo microscopy has been demonstrated with grid illumination in both an endomicroscope setup, ${ }^{12}$ and in conjunction with light-sheet illumination. ${ }^{13}$ While this freedom of choice of illumination patterns opens the possibility of using incoherent light sources, the benefits of coherent speckle illumination remain incontrovertible. The generation of speckle illumination is effortless as it requires essentially no imaging optics. Moreover, by dint of its being coherent, speckle retains its exceptionally high contrast completely independent of tissue scattering or aberrations. As such, HiLo microscopy with speckle illumination is simple, robust, and versatile. These qualities make HiLo microscopy useful for a broad array of bioimaging applications.

\section{Acknowledgments}

This was was supported by the NIH (R01 EB010059), the Coulter Foundation, and the Boston University Photonics Center. The authors thank Jinhyun Kim, Gal Haspel, and Dongdong Ma for providing samples.

1. J. B. Pawley, Handbook of Biological Confocal Microscopy, Springer, Berlin (2006).

2. W. Denk, J. H. Strickler, and W. W. Webb, "Two-photon laser scanning fluorescence microscopy," Science 248, 73-76 (1990).

3. M. A. A. Neil, T. Wilson, and R. Juskaitis "Methods of obtaining optical sectioning by using structured light in a conventional microscope," Opt. Lett. 22, 1905-1907 (1997). 
4. P. J. Verveer, Q. S. Hanley, P. W. Verbeek, L. J. van Vliet, and T. M. Jovin "Theory of confocal fluorescence imaging in the programmable array microscope. (PAM)," J. Microsc. 189 195-198 (1998).

5. J. Huisken J. Swoger, F. Del Bene, J. Wittbrodt, and E. H. K. Stelzer "Optical sectioning deep inside live embryos by selective plane illumination microscopy," Science 305, 1007-1009 (2004).

6. H.-U. Dodt, U. Leischner, A. Schierloh, N. Jährling, C. P. Mauch, K. Deininger, J. M. Deussing, M. Eder, W. Zieglgänsberger, and $\mathrm{K}$. Becker, "Ultramicroscopy: three-dimensional visualization of neuronal networks in the whole mouse brain," Nat. Methods 4, 331-336 (2007).

7. C. Ventalon and J. Mertz, "Quasi-confocal fluorescence sectioning with dynamic speckle illumination," Opt. Lett. 30, 3350-3352 (2005).

8. C. Ventalon and J. Mertz "Dynamic speckle illumination microscopy with translated versus randomized speckle patterns," Opt. Exp. 14, 7198-7209 (2006).

9. C. Ventalon, R. Heintzmann, and J. Mertz "Dynamic speckle illumination microscopy with wavelet prefiltering," Opt. Lett. 32, 1417-1419 (2007).

10. J. W. Goodman, Speckle Phenomena in Optics, Roberts and Company Publishers (2006).

11. D. Lim, K. K. Chu, and J. Mertz, "Wide-field fluorescence sectioning with hybrid speckle and uniform-illumination microscopy," Opt. Lett. 33, 1819-1821 (2008).

12. S. Santos, K. K. Chu, D. Lim, N. Bozinovic, T. N. Ford, C. Hourtoule, A. C. Bartoo, S. K. Singh, and J. Mertz, "Optically sectioned fluorescence endomicroscopy with hybrid-illumination imaging through a flexible fiber bundle," J. Biomed. Opt. 14, 030502 (2009).

13. J. Mertz and J. Kim "Scanning light-sheet microscopy in the whole mouse brain with HiLo background rejection," J. Biomed. Opt. 15 016027 (2010).
14. L. Leushacke and M. Kirchner "Three-dimensional correlation coefficient of speckle intensity for rectangular and circular apertures," J. Opt. Soc. Am. A 7, 827-832 (1990).

15. P. Stokseth, "Properties of a defocused optical system," J. Opt. 59, 1314-1321 (1969).

16. J. Mertz, Introduction to Optical Microscopy, Roberts and Company Publishers (2009).

17. I. Navarro-Quiroga, R. Chittajalu, V. Gallo, and T. F. Haydar, "Longterm, selective gene expression in developing and adult hippocampal pyramidal neurons using focal in utero electroporation," J. Neurosci. 27, 5007-5011 (2007).

18. C. J. R. Sheppard, M. Gu, K. Brain, and H. Zhou "Influence of spherical aberration on axial imaging of confocal reflection microscopy," Appl. Opt. 33, 616-624 (1994).

19. R. Kerr, V. Lev-Ram, G. Baird, P. Vincent, R. Y. Tsien, and W. R. Schafer, "Optical imaging of calcium transients in neurons and pharyngeal muscle of C. elegans," Neuron 26, 583-594 (2000).

20. A. Miyawaki, O. Griesbeck, R. Heim, and R. Y. Tsien, "Dynamic and quantitative $\mathrm{Ca} 2+$ measurements using improved cameleons," Proc. Natl. Acad. Sci. U.S.A. 96, 2135-2140 (1999).

21. R. M. White, A. Sessa, C. Burke, T. Bowman, J. LeBlanc, C. Ceol, C. Bourque, M. Dovey, W. Goessling, C. E. Burns, and L. I. Zon, "Transparent adult zebrafish as a tool for in vivo transplantation analysis," Cell Stem Cell 2, 183-189 (2008).

22. G. F. Marshall, Ed., Handbook of Optical and Laser Scanning, Marcel Dekker Inc., New York (2009).

23. H.-F. Lin, D. Traver, H. Zhu, K. Dooley, B. H. Paw, L. I. Zon, and R. I. Handin, "Analysis of thrombocyte development in CD41-GFP transgenic zebrafish," Blood 106, 3803-3810 (2005).

24. R. K. Jain, L. L. Munn, and D. Fukumura "Dissecting tumour pathophysiology using intravital microscopy," Nature Rev. Cancer 2, 266$276(2002)$. 